\title{
Composite porosity characterization using $x$-ray edge illumination phase contrast and ultrasonic techniques
}

Shoukroun, D., Massimi, L., Endrizzi, M., Bate, D., Fromme, P., et al.

D. Shoukroun, L. Massimi, M. Endrizzi, D. Bate, P. Fromme, A. Olivo, "Composite porosity characterization using $\mathrm{x}$-ray edge illumination phase contrast and ultrasonic techniques," Proc. SPIE 11593, Health Monitoring of Structural and Biological Systems XV, 115932M (22 March 2021); doi: $10.1117 / 12.2582561$

SPIE. Event: SPIE Smart Structures + Nondestructive Evaluation, 2021, Online Only 


\title{
Composite Porosity Characterization using X-ray Edge Illumination Phase Contrast and Ultrasonic Techniques
}

\author{
D. Shoukroun*a,c ${ }^{*}$ L. Massimi ${ }^{\text {a }}$, M. Endrizzi ${ }^{a}$, D. Bate ${ }^{b}$, P. Fromme ${ }^{c}$, A. Olivo $^{\text {a }}$ \\ ${ }^{a}$ Department of Medical Physics and Bioengineering, UCL, London, WC1E 7JE, UK \\ b Nikon, X-Tek Systems Ltd., Tring, UK \\ ${ }^{c}$ Department of Mechanical Engineering, UCL, London, WC1E 7JE, UK \\ *dana.shoukroun.17@ucl.ac.uk
}

\begin{abstract}
Owing to their combination of low weight and high strength, carbon fiber reinforced composites are widely used in the aerospace industry, including for primary aircraft structures. Porosity introduced by the manufacturing process can compromise structural performance and integrity, with a maximum porosity content of $2 \%$ considered acceptable for many aerospace applications. The main nondestructive evaluation (NDE) techniques used in industry are ultrasonic imaging and X-ray computed tomography, however both techniques have limitations. Edge Illumination X-ray Phase Contrast Imaging (EI XPCi) is a novel technique that exploits the phase effects induced by damage and porosity on the X-ray beam to create improved contrast. EI XPCi is a differential (i.e., sensitive to the first derivative of the phase), multi-modal phase method that uses a set of coded aperture masks to acquire and retrieve the absorption, refraction, and ultra-small-angle scattering signals, the latter arising from sub-pixel sample features. For carbon fiber-reinforced woven composite specimens with varying levels of porosity, porosity quantification obtained through various signals produced by EI XPCi was compared to ultrasonic immersion absorption C-scans and matrix digestion. The standard deviation of the differential phase is introduced as a novel signal for the quantification of porosity in composite plates, with good correlation to ultrasonic attenuation.
\end{abstract}

Keywords: CFRP, XPCi, Immersion Ultrasound Attenuation, Porosity

\section{INTRODUCTION}

Porosity is defined as a large number of sub-millimeter voids that do not affect the structural integrity of the plate on an individual level, but can affect the mechanical properties of the sample as a whole [1]. Porosity occurs during the manufacturing process of composites, and the aim is to minimize it or keep below $2 \%$ according to the current industrial standards $[1,2]$. Different techniques are used for porosity estimation in composite materials, destructive methods include matrix digestion [3] and micrography [4]. For the non-destructive evaluation (NDE) of porosity, ultrasonic imaging [5-7] and X-ray computed tomography (CT) [8-10] are the standard techniques employed in industry. Ultrasonic imaging is practical and can be used in situ, but has relatively low resolution $(100 \mu \mathrm{m})$. X-ray CT allows for 3D visualization and, assuming sufficient resolution, can produce a porosity map of the entire scanned sample, however it has limitations in sample size and typically requires long scanning times.

EI XPCi is a new X-ray imaging technique that relies on the phase effects that accompany the absorption of X-rays when they pass through a material. In conventional X-ray imaging, variations in contrast rely on changes in the material attenuation coefficient and on the thickness of the feature of interest. For very thin features, or features with similar attenuation coefficients to their surroundings, conventional X-ray imaging often provides insufficient contrast, while XPCi allows a better contrast as it relies on the real part of the refractive index. When an object is introduced into an $\mathrm{X}$-ray beam, the X-ray wavefront is distorted, causing a change in the direction of wave propagation [11]. Different XPCi methods exist, such as Talbot Lau interferometry [12] and free space propagation [13]. EI XPCi is a differential phase imaging technique, meaning that it measures the change in the refraction angle of the X-ray as it changes direction of propagation $[14,15]$; this refraction angle is proportional to the first derivative of the phase change. This is done using a set of coded aperture masks to convert the phase effects into a change in the detected intensity [15]. EI XPCi allows for the simultaneous acquisition and retrieval of the absorption, refraction, and ultra-small angle scattering signals, the latter

Health Monitoring of Structural and Biological Systems XV, edited by Paul Fromme,

Zhongqing Su, Proc. of SPIE Vol. 11593, 115932M - (c) 2021 SPIE

CCC code: $0277-786 \mathrm{X} / 21 / \$ 21 \cdot$ doi: $10.1117 / 12.2582561$

Proc. of SPIE Vol. 11593 115932M-1 
due to features in the sub-pixel scale, with the acquisition of at least three images [16]. EI XPCi is robust against environmental and energy variation, and can be implemented in a laboratory environment with a polychromatic and divergent beam, with variations of focal spot and pixel size having a manageable effect on its sensitivity [11]. Various XPCi methods were previously used to investigate impact damage [13, 17-19] and porosity [20,21], and previous work has demonstrated the viability of EI XPCi as an NDE method for impact damage detection in composite plates [22, 23]. The complementarity offered by the phase-based signals was demonstrated to be effective in the determination of the type of damage present in the sample due to the different ranges of features observed in each channel. As a result, the refraction signal was demonstrated to highlight the presence of delaminations in samples, whereas the scattering signal proved effective in showing the full extent of the damage by highlighting sub-pixel features [23].

In this investigation, EI XPCi is used for the quantification of porosity in fiber reinforced woven composite plates by comparing the three channels (absorption, refraction, and scattering) with ultrasonic through transmission immersion Cscan imaging. A new signal, the standard deviation of the refraction signal, is introduced as a viable measure for the quantification of porosity content in composite plates, by comparing it to ultrasonic attenuation. The accuracy of the ultrasonic measurement is tested by comparing the attenuation signals to porosity values calculated from matrix digestion.

\section{METHODOLOGY}

A set of carbon fiber reinforced woven composite specimens were manufactured at the University of Manchester, containing varying levels of porosity, ranging from $0.7 \%$ to $10.7 \%$. Nine plates of dimensions $120 \mathrm{~mm} \times 160 \mathrm{~mm}$ were manufactured with a layup consisting of 10 pre-preg M21 epoxy-carbon woven fabric plies. The specimens were extracted from the plates that underwent a standard autoclave cure recommended for M21 laminates by Hexcel [24], which included the following parameters: panels were pressurized to $7 \mathrm{bar}$, heated at $2^{\circ} \mathrm{C} / \mathrm{min}$; cure dwell at $180^{\circ} \mathrm{C}$ for $120 \mathrm{~min}$, cooling at $5^{\circ} \mathrm{C} / \mathrm{min}$. Panels were depressurized when the temperature dropped below $60^{\circ} \mathrm{C}$, with $100 \%$ vacuum for the whole duration of the cure. Variations of the cure parameters were performed for each plate (consolidation of laminates by vacuum debulk during lay-up, edge breathing of laminate in vacuum bag, time under vacuum prior to start of cure, applied pressure during cure and cure temperature profile). Nine specimens of dimensions $100 \mathrm{~mm}$ x $50 \mathrm{~mm}$ were extracted from the plates after cure. Three additional $20 \mathrm{~mm} \times 10 \mathrm{~mm}$ samples were extracted from areas in the plates next to the extracted specimen to determine the porosity content of the plates using matrix digestion, assuming nominal densities of $1.78 \mathrm{~g} / \mathrm{cm}^{3}$ for the fibers and $1.28 \mathrm{~g} / \mathrm{cm}^{3}$ for the matrix [25]. The variations in autoclave cure processes resulted in specimens with different porosity content, described in Table 1.

For X-ray imaging, $15 \mathrm{~mm} \times 15 \mathrm{~mm}$ regions of interest (ROI) were delimited on the edges of the plates using reflective tape, as shown in Fig. 1, due to the limited field of view of the X-ray system used and the need to include a background region in the scan for image normalization. As a result, $75 \%$ of the plate was scanned using X-rays, split into 18 ROIs, which were covered with 10 separate X-ray acquisitions.

Table 1. Porosity values calculated from matrix digestion of the nine manufactured specimens.

\begin{tabular}{|l|l|l|l|l|l|l|l|l|l|}
\hline Porosity (\%) & $10.7 \%$ & $6.6 \%$ & $5.9 \%$ & $3.9 \%$ & $1.5 \%$ & $1.3 \%$ & $0.9 \%$ & $0.9 \%$ & $0.7 \%$ \\
\hline
\end{tabular}

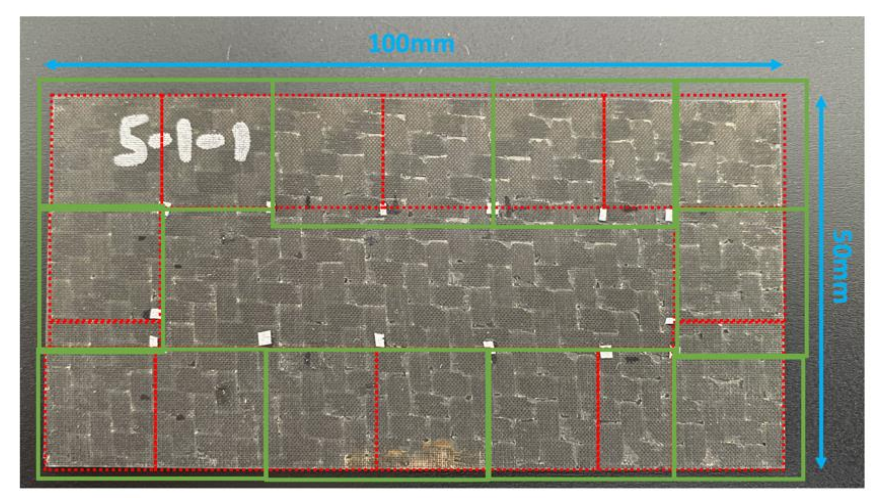

Figure 1. Specimen used for porosity investigation, with $15 \mathrm{~mm}$ x $15 \mathrm{~mm}$ ROIs marked in red and the areas covered by the $\mathrm{X}$-ray acquisitions delimited in green. 


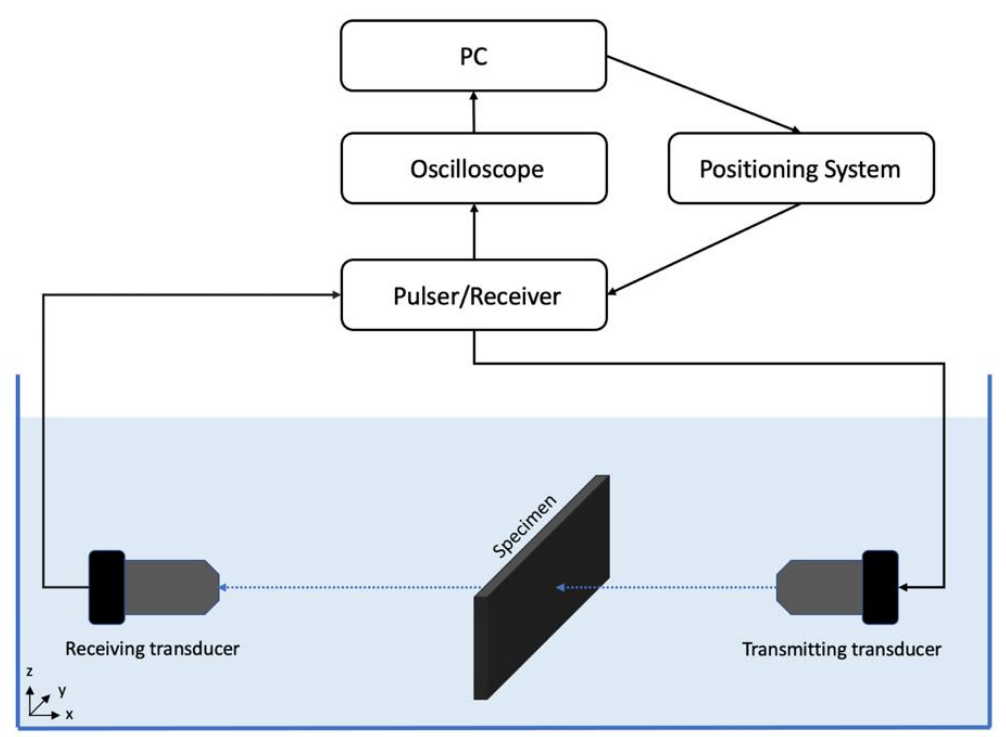

Figure 2. Setup used for the through transmission ultrasonic C-scan imaging of the porosity specimens.

The specimens were scanned using ultrasonic through transmission C-scan imaging at two different frequencies for comparison. The first scan included a $2.25 \mathrm{MHz}$ focused emitting transducer (Olympus U8420169), with 0.25 in (6 mm) nominal diameter, $0.4 \mathrm{in}(10 \mathrm{~mm})$ focal length, $1.5 \mathrm{~mm}$ focal spot and $670 \mu \mathrm{m}$ wavelength. The second scan used a 2.5 $\mathrm{MHz}$ unfocussed emitting transducer, with $20 \mathrm{~mm}$ nominal diameter, a near field of $167 \mathrm{~mm}$ and $600 \mu \mathrm{m}$ wavelength. For both measurements a $2.5 \mathrm{MHz}$ unfocussed receiving transducer was used. The setup used is shown in Fig. 2. The transmitting transducer, immersed in water and connected to a pulser receiver (Panametrics 5601T), emits a pulse that is transmitted through the sample. The transmitted signal is captured by the receiving transducer, also connected to the pulser/receiver. The output signal is recorded by an Oscilloscope (LeCroy 9304) and the resulting signal saved as an Ascan. The scans using the unfocussed $2.5 \mathrm{MHz} /$ unfocussed $2.5 \mathrm{MHz}$ transducer pair were performed using a $2 \mathrm{~mm}$ step size, resulting in 61 by 31 steps required to cover the full plate. The scans with the focused $2.25 \mathrm{MHz} /$ unfocussed $2.5 \mathrm{MHz}$ transducer pair was done with a $1 \mathrm{~mm}$ step size, resulting in 121 by 61 steps. The A-scans were used to calculate the average signal attenuation of the ultrasonic signal by comparing it with the average signal emitted in water, using the equation:

$$
\Delta \mathrm{I}(\mathrm{dB})=20 \log \left(\mathrm{V}_{\text {water }} / \mathrm{V}_{\text {plate }}\right)
$$

where $\Delta \mathrm{I}$ is the attenuation of the signal in $\mathrm{dB}, \mathrm{V}_{\text {water }}$ is the maximum amplitude of the ultrasonic signal in water, and $\mathrm{V}_{\text {plate }}$ is the maximum amplitude of the ultrasonic signal through the specimen

The experimental setup for the Edge Illumination (EI XPCi) system is shown in Fig. 3. The first ("sample") mask is placed upstream of the sample and splits the beam into individual beamlets. The "detector" mask, placed adjacent to the detector, makes the area between adjacent pixel insensitive to incoming X-rays. When a sample is placed in the beam, the X-rays change their direction of propagation due to refraction [26]. The used system [27] included a Rigaku MicroMax $007 \mathrm{HF}$ rotating anode molybdenum source with a $70 \mu \mathrm{m}$ focal spot, operating at $40 \mathrm{kVp}$ and $20 \mathrm{~mA}$. The detector was an indirect flat panel CMOS detector with a $50 \times 50 \mu \mathrm{m}^{2}$ pixel size. The sample mask, placed $0.7 \mathrm{~m}$ from the source, had an aperture of $12 \mu \mathrm{m}$ and a pitch of $78 \mu \mathrm{m}$; the detector mask, placed $0.85 \mathrm{~m}$ from the source, had an aperture of $20 \mu \mathrm{m}$ and a pitch of $98 \mu \mathrm{m}$. The detector mask was a skipped mask, meaning that every other pixel was made insensitive to incoming X-rays. This was done in order to reduce the cross-talk between neighboring pixels [28]. The system was only sensitive to phase effect in the $\mathrm{x}$-direction. The image acquisition process included the acquisition of the illumination curve (IC), which is a bell-shaped curve that represents the variation of detected intensity at different positions of the sample mask relative to the detector masks. This was done by acquiring 19 flat field images at 19 relative sample mask positions, one at the position where the two masks are perfectly aligned, and 9 at symmetric, increasing displacements in each direction. 


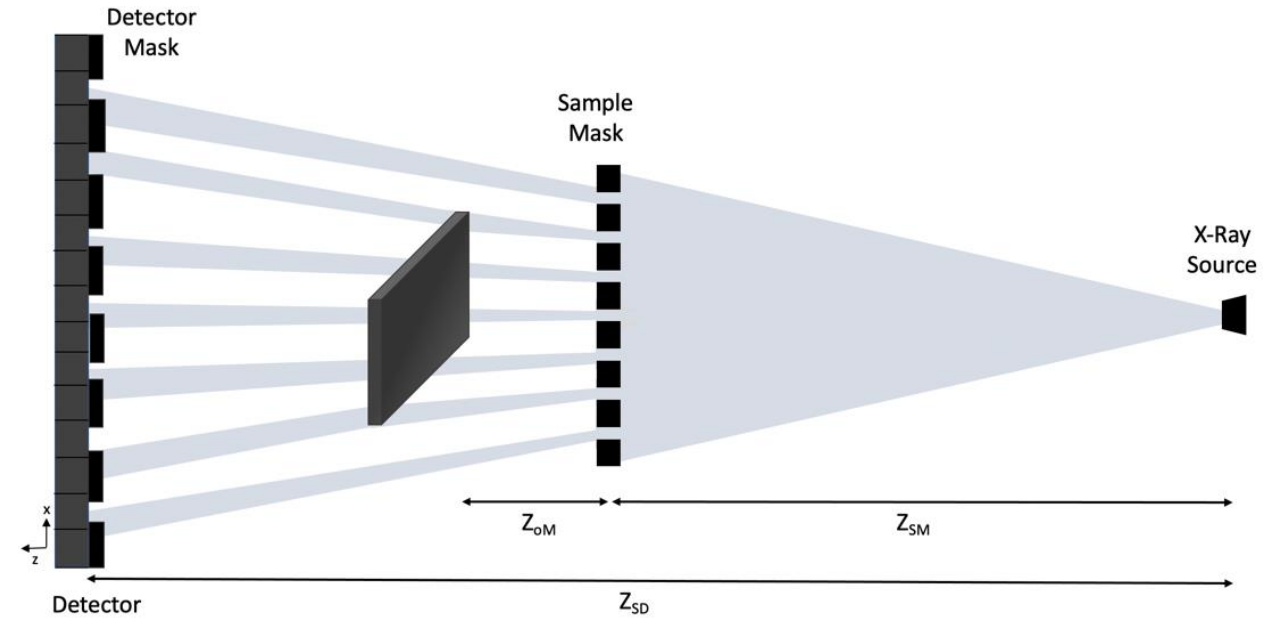

Figure 3. Laboratory setup of the edge illumination phase contrast imaging system.

The samples were then introduced, and another 19 images were acquired for each specimen with the same relative sample mask positions as in the IC case. All images were acquired with $6 \mathrm{~s}$ exposure time. To increase image resolution, the sample was dithered (i.e., repositioned in different sub-pixel locations) 16 times. This allowed to reach a resolution determined by the sample mask aperture, i.e. $12 \mu \mathrm{m}$, in the $\mathrm{x}$-direction, while resolution in the $\mathrm{y}$-direction remains dictated by the detector performance (i.e. $100 \mu \mathrm{m}$, [29]). The phase retrieval of the three signals is based on the method described in [30], where the illumination curve is assumed to have a Gaussian shape. Different object-induced modifications of the Gaussian correspond to the three contrast channels provided by EI XPCi: absorption causes changes in its amplitude, refraction leads to a shift in its central position, and scattering leads to a broadening of the curve. The phase retrieval process is based on fitting a Gaussian to the illumination curve with and without a sample, and comparing the three parameters described above on a pixel-by-pixel basis to extract the corresponding contrast channels. An additional signal is introduced in this investigation, the standard deviation of the refraction signal, which corresponds to the presence of a number of object interfaces on a scale larger than the system resolution. This can be considered similar to the scattering signal for features equal to or larger than the system resolution, and therefore capable of detecting variations in the distribution of sample inhomogeneities on a scale larger than $12 \mu \mathrm{m}$. Note that, since the system is only sensitive to phase effects along the $\mathrm{x}$-direction, refraction (or scattering, for that matter) signals are not detected along the $\mathrm{y}$-direction, which makes resolution along that direction irrelevant to the analysis conducted here.

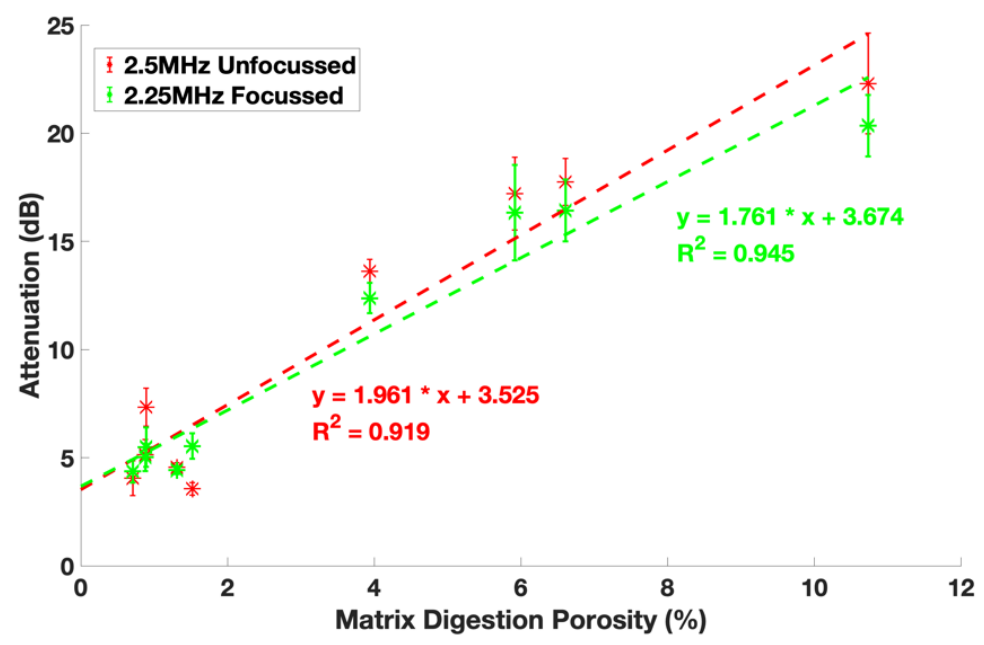

Figure 4. Comparison of ultrasonic signal attenuation with porosity values obtained from matrix digestion for nine specimens, using focussed $(2.25 \mathrm{MHz})$ and unfocussed $(2.5 \mathrm{MHz})$ transducers. 


\section{RESULTS}

The first step in this investigation was to compare the average signal attenuation of the ultrasonic signal across the full plates from both the focused and unfocussed transducers to the porosity values obtained from the matrix digestion. This comparison can be seen in Fig. 4. Both attenuation values measured using the $2.5 \mathrm{MHz}$ unfocussed and $2.25 \mathrm{MHz}$ focused transducers show a very good correlation with the porosity content, especially for high porosities. It can be seen that, for low porosities $(<2 \%)$, the ultrasonic signal shows little variation with the porosity value.

Each plate generated 18 data points in each of the three X-ray signals, corresponding to the 18 ROIs defined (Fig. 1). The same ROIs were also delimited in the ultrasonic signals, and the average ultrasonic signal attenuation was calculated for each ROI to allow for a comparison with the X-ray signals. The three signals obtained from the EI XPCi system were plotted against the ultrasonic attenuation signal from both focused and unfocussed transducers. As an example, the absorption and scattering signals are shown in Fig. 5 for the plate with a matrix digestion porosity of 5.9\%.

Some correlation can be seen between the absorption signal and ultrasonic attenuation; however, the large error bars indicate that the signal variation of the X-ray absorption signal is small compared to the variation in ultrasonic attenuation, both for the focused and unfocussed transducers. The variation observed here could indicate variations in the thickness of the plate, due to a possible uneven distribution of epoxy during the curing process, as opposed to different levels of porosity in different areas of the plate. The X-ray scattering signal was also plotted against the ultrasonic signal attenuation from both transducers. Here, no correlation can be observed. Since scattering was proven to be highly effective in the detection of microscopic damage [23], we attribute this to the lack of porosity features on the relevant scale, i.e., below $12 \mu \mathrm{m}$.
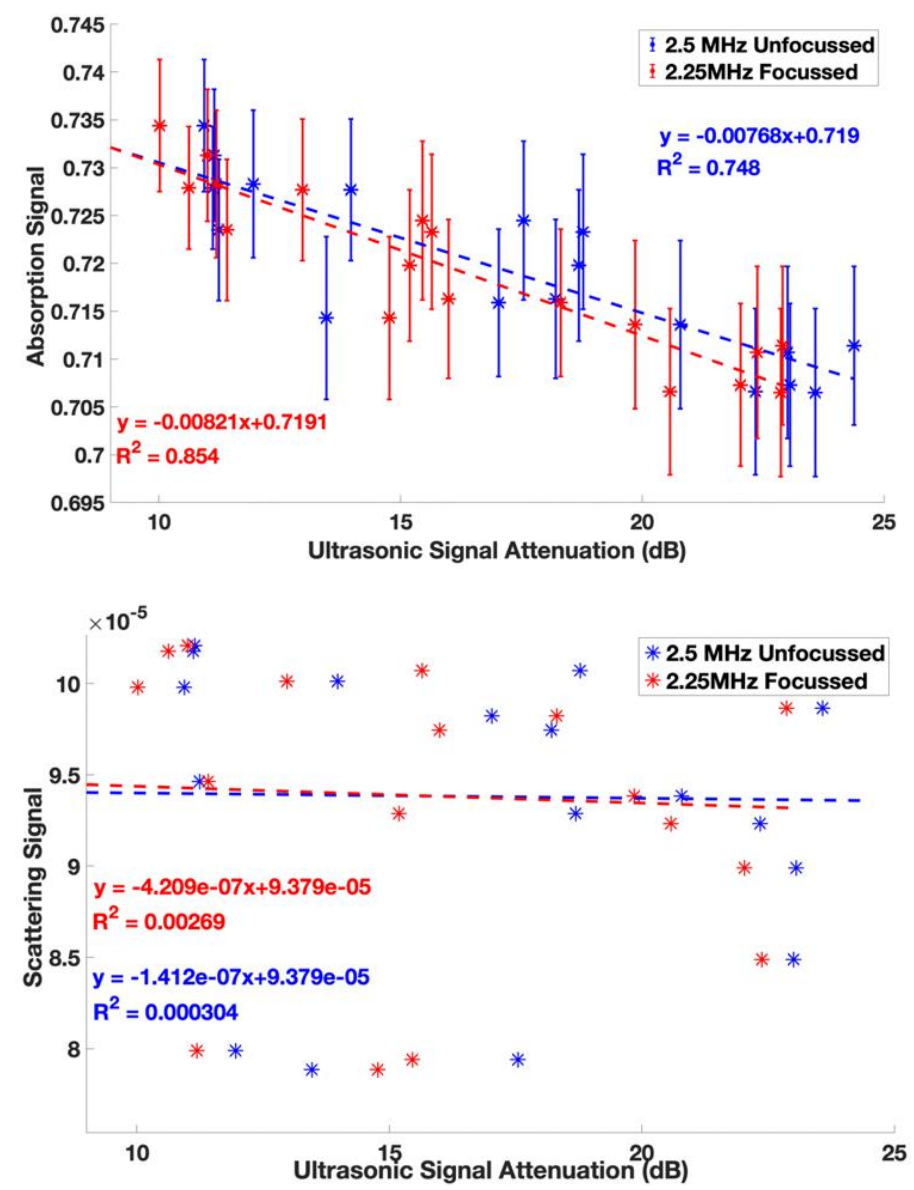

Figure 5. Comparison of the absorption and scattering signals obtained from the EI XPCi image with both the $2.5 \mathrm{MHz}$ unfocussed and $2.25 \mathrm{MHz}$ focused transducers for the specimen with $5.9 \%$ porosity. 


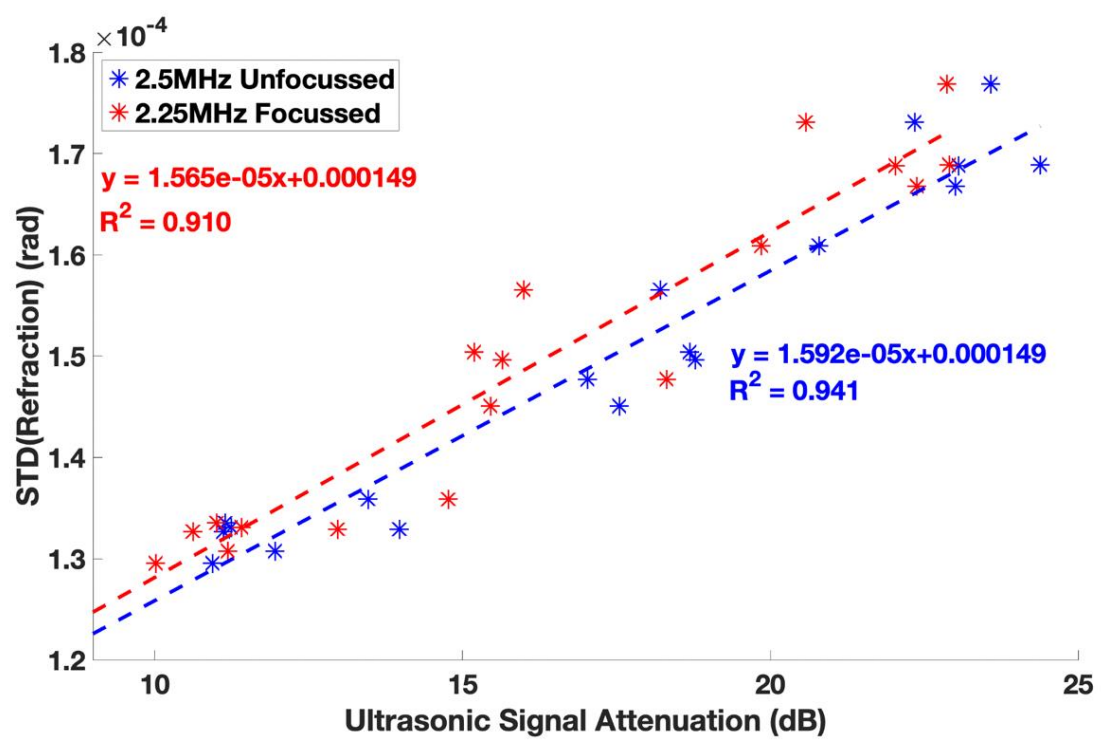

Figure 6. Standard deviation of the refraction signal compared with ultrasonic signal attenuation for the $2.5 \mathrm{MHz}$ unfocussed and $2.25 \mathrm{MHz}$ focused transducers for the specimen with $5.9 \%$ porosity.

In order to account for the possibility that the average size of the pores distributed across the $5.9 \%$ porosity sample could be larger than $12 \mu \mathrm{m}$, the standard deviation of the refraction signal was compared with the ultrasonic attenuation signals for both transducers, as shown in Fig. 6. Note that it would have little meaning to perform a comparison directly with the refraction signal itself, as this simply highlights the edges of the features. For this reason, its standard deviation expresses how many "edges" on average are present within a certain region, thus providing a similar signal to the scattering channel, but over larger (i.e., resolvable) signals. As can be seen, a strong correlation is observed, both with the unfocussed and the focused transducers. This indicated that the standard deviation of the refraction signal can be used as a tool for the quantification of porosity in composite plates when the average size of the defects exceeds the system spatial resolution.

The same analysis was performed on all nine plates, and the standard deviation of the refraction signal was plotted against the ultrasonic signal attenuation for the 18 ROIs of each specimen, for the ultrasonic attenuation values obtained both the unfocussed and focused transducer. The results are shown in Fig. 7.
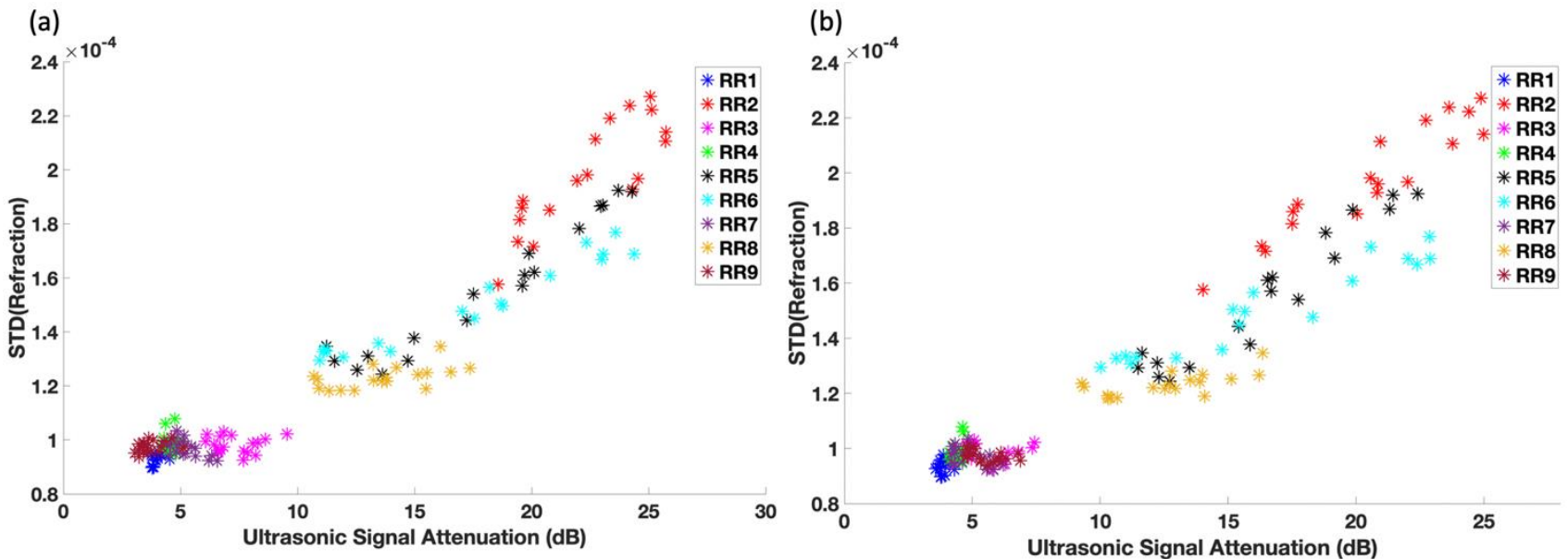

Figure 7. Standard deviation of the refraction signal for 18 ROIs for the 9 specimens compared with ultrasonic signal attenuation for: (a) $2.5 \mathrm{MHz}$ unfocussed transducer; (b) $2.25 \mathrm{MHz}$ focused transducer. 
A clear correlation can be observed between the standard deviation of the refraction and the ultrasonic attenuation signal obtained using both the unfocussed and the focused transducers. The correlation fits best for plates with ultrasonic signal attenuation above $10 \mathrm{~dB}$, which corresponds to plates with porosity above $2 \%$. For plates below $2 \%$ porosity, only limited variation in signal is observed in the standard deviation of the refraction for the individual ROIs, whereas the ultrasonic signal attenuation seems to vary more. However, as shown in Fig. 4, the ultrasonic attenuation does not correlate well with porosity from matrix digestion for plates below $2 \%$ porosity. While from these results one could be tempted to conclude that the standard deviation of the refraction signal can be used as a tool for the quantitative estimation of porosity for plates with at least $2 \%$ porosity, in truth the interplay between scatter and standard deviation of the refraction signal must be understood in more detail before firm conclusions can be drawn.

\section{CONCLUSIONS}

Ultrasonic signal attenuation measured using transducers with different frequencies and foci was compared for carbon fiber reinforced woven composite plates with different porosity, calculated from matrix digestion. It was found that the ultrasonic signal attenuation correlates with porosity content for porosities above $2 \%$. Below $2 \%$, the signal attenuation did not vary significantly with varying porosity. The use of a focused transducer gave a slightly better correlation with porosity than unfocussed transducers, an expected result as the focused beam allows for a better spatial resolution and thus better resolution of the porosity in the samples.

Edge illumination (EI XPCi) was used for the quantification of porosity in the composite plates by comparison with ultrasonic attenuation. Specific ROIs were compared for each individual plate. Limited correlation was found between the ultrasonic attenuation and the absorption and scattering contrast channels. A new signal, the standard deviation of the refraction, which represents the variation in inhomogeneity distribution across the ROI for features on a scale equal to or above the system resolution, was introduced for the quantification of porosity. A good correlation was found between the ultrasonic attenuation and the standard deviation of the refraction for plates with porosity levels above $2 \%$. Further investigations will be required to assess whether the limited correlation below $2 \%$ is due to the nature of the new signal or to limitations of the ultrasonic attenuation measurements, since ultrasonic attenuation did not correlate with the matrix digestion porosity values below $2 \%$. Moreover, the interplay between the standard deviation of the refraction and scattering must be further investigated, as the simultaneous presence of pores above and below the system resolution may require some complex weighting between the two signals to produce a reliable quantification.

\section{REFERENCES}

[1] E. A. Birt and R. A. Smith, "A review of NDE methods for porosity measurement in fibre-reinforced polymer composites," Insight Non-Destructive Test. Cond. Monit., vol. 46, no. 11, pp. 681-686, 2004, doi: 10.1784/insi.46.11.681.52280.

[2] G. Fernlund, J. Wells, L. Fahrang, J. Kay, and A. Poursartip, "Causes and remedies for porosity in composite manufacturing," IOP Conf. Ser. Mater. Sci. Eng., vol. 139, no. 1, 2016, doi: 10.1088/1757-899X/139/1/012002.

[3] M. H. Hassan, A. R. Othman, and S. Kamaruddin, "Void content determination of fiber reinforced polymers by acid digestion method," Adv. Mater. Res., vol. 795, 2013, doi: 10.4028/www.scientific.net/AMR.795.64.

[4] A. H. Kite, D. K. Hsu, and D. J. Barnard, "Determination of porosity content in composites by micrograph image processing," AIP Conf. Proc., vol. 975, pp. 942-949, 2008, doi: 10.1063/1.2902767.

[5] H. Jeong, "Effects of Voids on the Mechanical Strength and Ultrasonic Attenuation of Laminated Composites," J. Compos. Mater., vol. 31, no. 3, pp. 276-292, 1997.

[6] R. D. Adams and P. Cawley, "A review of defect types and nondestructive testing techniques for composites and bonded joints," NDT E Int., vol. 21, no. 4, p. 105, 1988, doi: 10.1016/0963-8695(91)90924-R.

[7] I. M. Daniel, S. C. Wooh, and I. Komsky, "Quantitative porosity characterization of composite materials by means of ultrasonic attenuation measurements," J. Nondestruct. Eval., vol. 11, no. 1, pp. 1-8, 1992, doi: 10.1007/BF00566012.

[8] P. Yang and R. Elhajjar, "Porosity Content Evaluation in Carbon-Fiber/Epoxy Composites Using X-ray Computed Tomography," Polym. - Plast. Technol. Eng., vol. 53, no. 3, pp. 217-222, 2014, doi: 10.1080/03602559.2013.843700.

[9] A. Reh, B. Plank, J. Kastner, E. Gröller, and C. Heinzl, "Porosity maps - Interactive exploration and visual analysis of porosity in carbon fiber reinforced polymers," Comput. Graph. Forum, vol. 31, no. 3, pp. 1185-1194, 2012, doi: 10.1111/j.1467-8659.2012.03111.x. 
[10] E. Maire and P. J. Withers, “Quantitative X-ray tomography,” Int. Mater. Rev., vol. 59, no. 1, pp. 1-43, 2014, doi: 10.1179/1743280413Y.0000000023.

[11] A. Olivo and E. Castelli, "X-Ray Phase Contrast Imaging: From Synchrotrons to Conventional Sources," Riv. del Nuovo Cim., vol. 37, no. 9, pp. 467-508, 2014, doi: 10.1393/ncr/i2014-10104-8.

[12] V. Revol, B. Plank, R. Kaufmann, J. Kastner, C. Kottler, and A. Neels, "Laminate fibre structure characterisation of carbon fibre-reinforced polymers by X-ray scatter dark field imaging with a grating interferometer," NDT E Int., vol. 58, pp. 64-71, 2013, doi: 10.1016/j.ndteint.2013.04.012.

[13] S. C. Mayo, A. W. Stevenson, and S. W. Wilkins, "In-Line Phase-Contrast X-ray Imaging and Tomography for Materials Science,” Materials (Basel)., vol. 5, no. 12, pp. 937-965, 2012, doi: 10.3390/ma5050937.

[14] A. Olivo and R. Speller, "Modelling of a novel x-ray phase contrast imaging technique based on coded apertures," Phys. Med. Biol., vol. 52, no. 22, pp. 6555-6573, 2007, doi: 10.1088/0031-9155/52/22/001.

[15] A. Olivo and R. Speller, "Image formation principles in coded-aperture based x-ray phase contrast imaging," Phys. Med. Biol., vol. 53, no. 22, pp. 6461-6474, 2008, doi: 10.1088/0031-9155/53/22/012.

[16] M. Endrizzi et al., "Hard X-ray dark-field imaging with incoherent sample illumination," Appl. Phys. Lett., vol. 104, no. 2, 2014, doi: 10.1063/1.4861855.

[17] P. Cloetens et al., "Observation of microstructure and damage in materials by phase sensitive radiography and tomography,” J. Appl. Phys., vol. 81, no. 5878, 1997, doi: 10.1063/1.364374.

[18] O. Coindreau, G. Vignoles, and J.-M. Goyheneche, "Multiscale X-Ray CMT of C/C Composite Preforms: A Tool for Properties Assessment," in Ceramic Transactions, Advances in Ceramic Matrix Composites, 2006, vol. 11, pp. 77-84.

[19] F. Cosmi, A. Bernasconi, and N. Sodini, "Phase contrast micro-tomography and morphological analysis of a short carbon fibre reinforced polyamide," Compos. Sci. Technol., vol. 71, no. 1, pp. 23-30, 2011, doi: 10.1016/j.compscitech.2010.09.016.

[20] V. Revol et al., "Sub-Pixel Porosity Revealed by X-Ray Scatter Dark Field Imaging," J. Appl. Phys., vol. 110, no. 044912, pp. 1-5, 2011, doi: 10.1063/1.3624592.

[21] C. Gusenbauer, M. Reiter, B. Plank, D. Salaberger, S. Senck, and J. Kastner, "Porosity Determination of Carbon and Glass Fibre Reinforced Polymers Using Phase-Contrast Imaging," J. Nondestruct. Eval., vol. 38, no. 1, pp. 1-10, 2019, doi: 10.1007/s10921-018-0529-6.

[22] M. Endrizzi, B. I. S. Murat, P. Fromme, and A. Olivo, "Edge-illumination X-Ray Dark-Field Imaging for Visualising Defects in Composite Structures,” Compos. Struct., vol. 134, pp. 895-899, 2015, doi: 10.1016/j.compstruct.2015.08.072.

[23] D. Shoukroun et al., "Enhanced composite plate impact damage detection and characterisation using X-Ray refraction and scattering contrast combined with ultrasonic imaging," Compos. Part B Eng., vol. 181, no. 107579, 2020, doi: 10.1016/j.compositesb.2019.107579.

[24] "HexPly $®$ M21 180C Curing Epoxy Matrix Report," 2020.

[25] “ASTM D3171-15," in Standard Test Methods for Constituent Content of Composite Materials, West Conshohocken, PA: ASTM International, 2015.

[26] P. C. Diemoz et al., "X-ray Phase Contrast Imaging with Nanoradian Angular Resolution,” Phys. Rev. Lett., vol. 110, no. 13, pp. 1-5, 2013, doi: 10.1103/PhysRevLett.110.138105.

[27] L. Massimi et al., "Detection of involved margins in breast specimens with X - ray phase - contrast computed tomography," Sci. Rep., vol. 11, no. 3663, pp. 1-9, 2021, doi: 10.1038/s41598-021-83330-w.

[28] K. Ignatyev, P. R. T. Munro, R. D. Speller, and A. Olivo, "Effects of signal diffusion on x-ray phase contrast images," Rev. Sci. Instrum., vol. 82, no. 7, 2011, doi: 10.1063/1.3606442.

[29] P. C. Diemoz, F. A. Vittoria, and A. Olivo, "Spatial resolution of edge illumination X-ray phase-contrast imaging," Opt. Express, vol. 22, no. 13, p. 15514, 2014, doi: 10.1364/oe.22.015514.

[30] C. J. Maughan Jones, F. A. Vittoria, A. Olivo, M. Endrizzi, and P. R. T. Munro, "Retrieval of weak X-ray scattering using edge illumination," Opt. Lett., vol. 43, no. 16, p. 3874, 2018, doi: 10.1364/ol.43.003874. 